\title{
Effects of lactation on rate of blood ethanol disappearance, ethanol consumption, and serum electrolytes in the rat
}

\author{
E. L. ABEL \\ Research Institute on Alcoholism, Buffalo, New York 14203
}

\begin{abstract}
The rate of blood ethanol disappearance was faster in lactating compared with virgin female rats and was also faster in lactating females nursing 10 sucklings than in those nursing 2 sucklings. The increased rate of blood ethanol disappearance was not associated with an increased consumption of ethanol. Serum sodium levels were lower in lactating females, although fluid consumption was markedly increased.
\end{abstract}

During lactation, there is a change in the responsiveness of the nursing female to various drugs. For example, Peters and Leeuwin (1971) have observed that pentobarbital-induced "sleeping time" is shorter in lactating compared with nonlactating rats. Thiessen, Whitworth, and Rogers (1967) have demonstrated that the rate of blood ethanol disappearance is significantly faster in lactating $\mathrm{C}_{5}{ }_{7} \mathrm{~B} 1$ mice than in virgin female controls. We have corroborated the latter observation in rats and have also shown that the increased rate of blood ethanol disappearance does not occur in females whose litters are removed at birth, thus eliminating parturition as a possible factor in this observation (Greizerstein, Abel, \& Siemens, 1979). The present experiment was designed to investigate how changes in the size of the litter being nursed affect the rate of blood ethanol disappearance. A second experiment was also conducted to determine if the increased rate of blood ethanol disappearance is associated with an increased consumption of ethanol.

\section{METHOD}

Sprague-Dawley female rats approximately 5 months of age and weighing between 256 and $322 \mathrm{~g}$ at the time of study were used. On the day of delivery, litters were culled to 2,5 , or 10 pups per dam. Nulliparous females served as controls. On Day 7 postpartum for the lactating females, all animals were injected (intraperitoneally) with $3 \mathrm{~g} / \mathrm{kg}$ ethanol in $.9 \%$ saline $(20 \% \mathrm{~W} / \mathrm{V}, 15 \mathrm{ml} / \mathrm{kg})$. Litters were removed from dams at this time and were not returned until the end of the study. Beginning $2 \mathrm{~h}$ after injection, tail-tip blood samples (50 microliters) were taken from each rat at hourly intervals for the next $6 \mathrm{~h}$. Blood ethanol concentrations were subsequently determined according to the method of Greizerstein and Smith (1973), using propanol as an internal standard. Linear-regression equations were calculated for each animal by the method of least squares, using the 3- to 7-h blood concentration values, and these were then used to determine rates of blood ethanol disappearance.

I thank Dr. H. B. Greizerstein and Ms. P. Trumbull for gas chromatographic analysis.
In a second experiment, using a separate group of animals, litters were culled to eight pups per dam on the day of parturition, and dams were of fered a choice thereafter between either a $5 \% \mathrm{~V} / \mathrm{V}$ solution of ethanol in tap water or tap water only. Fluid consumption was determined by the difference in bottle weight for the virgin $(N=10)$ and lactating $(N=10)$ rats on a daily basis from Day 1 of parturition to Day 14. During this period, the position of the bottles was alternated after each daily determination of fluid intake. Food (Purina Lab Chow) was available ad lib.

On Day 13 postpartum, two additional groups of lactating and virgin females $(\mathrm{N}=10 /$ group) were sacrificed by decapitation, and blood samples were collected in heparinized tubes. These tubes were immediately centrifuged, the plasma was removed, and a short time later, the plasma was analyzed for sodium and potassium content using an Instrument Labs (IL 343) flame-photometer and for calcium using a Corning calcium titrator. Livers, kidneys, and spleens were also removed, trimmed of fat and connective tissue, and weighed.

\section{RESULTS}

The mean rates of blood ethanol disappearance ( \pm SE) were 293.8 (37.5) for virgin controls and 391.1 (19.2), $425.0(10.5)$, and $446.6(14.9) \mathrm{mg} / \mathrm{kg} / \mathrm{h}$ for lactating females nursing 2,5 , and 10 sucklings, respectively. Relative to the virgin females, these rates of blood ethanol disappearance are 133\%, 145\%, and $152 \%$ faster, respectively (see Figure 1).

A one-way analysis of variance indicated that these group differences were significant $[F(3,11)=11.79$, $\mathrm{p}<.005$ ] . Student's $\mathrm{t}$ tests indicated that the differences among the lactating groups nursing 2,5 , and 10 neonates and the virgin females were all significant $[t(7)=$ $3.59, \mathrm{t}(6)=4.75, \mathrm{t}(6)=5.12$, respectively; all ps $<.01]$. Among lactating animals, however, the only significant difference was between females nursing 2 and those nursing 10 sucklings $[\mathrm{t}(5)=2.28, \mathrm{p}<.05]$.

To minimize daily fluctuations in fluid consumption due to positional bias, the amount of fluids consumed daily were pooled for each 2 days of testing, and the data were examined on the basis of these 2-day values.

The amount of tap water and ethanol consumed 
in the two-choice situation for the lactating and virgin females is shown in Figure 2. As evident from the figure, lactating females consumed far more water than virgin females. By Days 13-14 postpartum, lactating females were consuming approximately $62 \mathrm{~g}$ of water daily, compared with approximately $15 \mathrm{~g}$ for virgin females. Although there was a trend for lactating females to consume more ethanol than virgins, differences between the two groups were not significant. Median ethanol preference scores [ethanol consumption/(ethanol + water consumption)] were .26 for lactating females and .47 for virgin females $(U=27, p=.05)$, indicating a lower overall preference for ethanol by the lactating animals.

Effect of lactation on blood electrolyte levels is presented in Table 1. Serum sodium levels were significantly lower in lactating animals compared with virgin controls $[\mathrm{t}(18)=2.12, \mathrm{p}<.05]$. Group differences for the other two electrolytes were not significant.

Mean body weights and weights of various internal organs for the two groups are also presented in Table 1. Compared with controls, lactating females had higher body, liver, and kidney weights. But when these organs were expressed as a function of body weight, only the difference in liver weights remained significantly higher for lactating females.

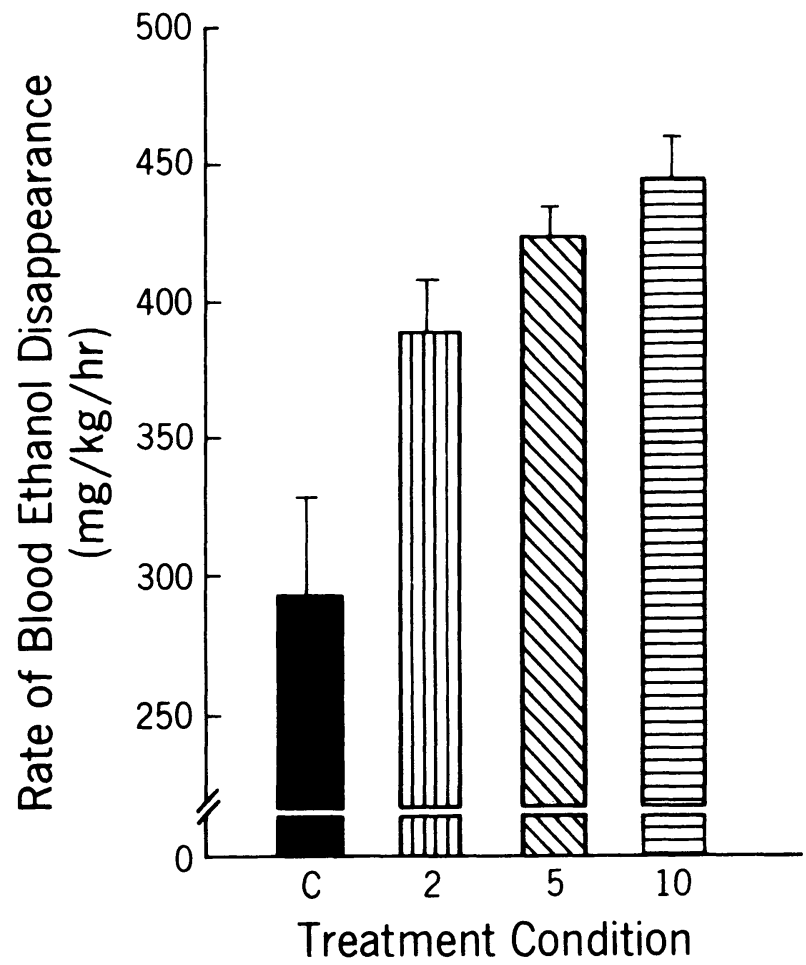

Figure 1. Rate of blood ethanol disappearance $(\mathrm{mg} / \mathrm{kg} / \mathrm{h})$ in Sprague-Dawley virgin female rats $(c)(N=5)$ and females nursing $2(\mathrm{~N}=4), 5(\mathrm{~N}=3)$, or $10(\mathrm{~N}=3)$ sucklings per litter.

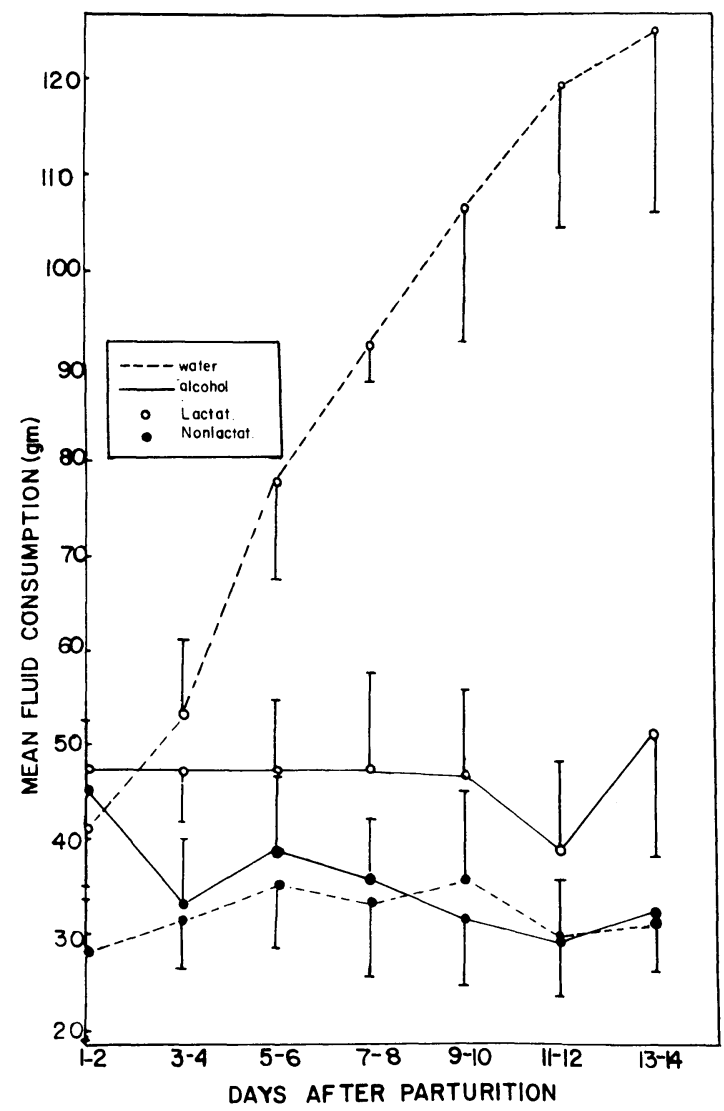

Figure 2. Mean 2-day water (-- ) and 5\% V/V alcohol $(-)$ intake of lactating $(0)$ and nonlactating $(\bullet)$ SpragueDawley female rats. $\mathrm{N}=10$ for each group. Vertical lines indicate standard error of the mean. The animals had both solutions available and the position of each bottle was alternated daily.

Table 1

Serum Electrolyte Levels (mEq/1), Body Weights, and Organ Weights of Lactating and Nonlactating Rats

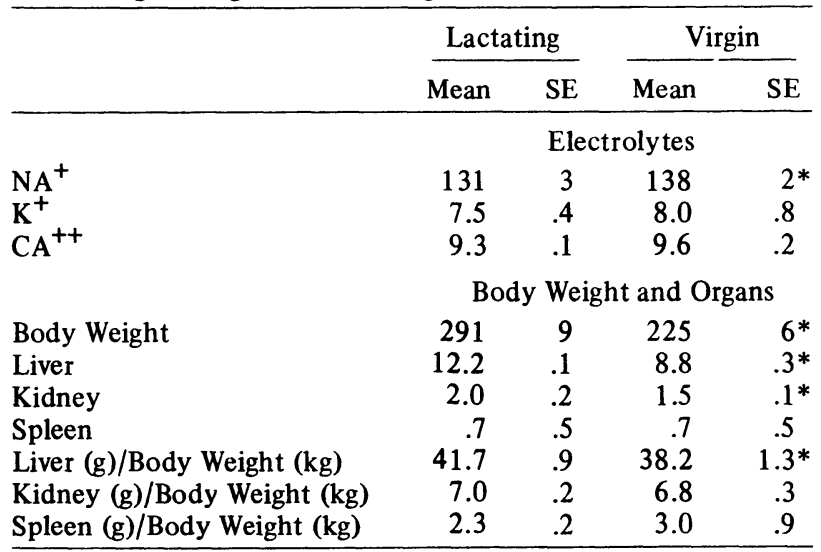

${ }^{*} p<.05$. 


\section{DISCUSSION}

These data corroborate previous studies demonstrating a more rapid rate of blood ethanol disappearance associated with lactation (Greizerstein et al., 1979; Thiessen et al., 1967). In addition, the present study demonstrates that the rate of blood ethanol disappearance is directly related to the intensity of sucking experienced by the mothers (i.e., the number of pups).

Although fluid intake increases markedly as nursing continues, this increase reflects a rather selective choice for water over a $5 \% \mathrm{~V} / \mathrm{V}$ ethanol solution. Although the nonlactating female does not exhibit a preference for either solution, it is clear that such a choice is made by the lactating female, and despite the increased rate of ethanol elimination by lactating animals, there is no preference for ethanol.

It is interesting that lactating females had significantly lower plasma sodium levels, since this condition could be expected to be associated with a decrease in fluid intake (cf. Corbet, 1965). However, this was obviously not the case.

The increased size of the liver relative to body weight observed in lactating females in the present study and in other studies (e.g., Bade \& Echave Llanos, 1963; Kennedy, Pearce, \& Parrott, 1958; Thiessen et al., 1967) suggests a possible basis for the increased rate of blood ethanol disappearance on the part of lactating animals, since this change may be associated with changes in functional metabolic activity (Thiessen et al., 1967).

\section{REFERENCES}

Bade, E. G., \& Echave Llanos, J. M. Increase in the weight of the liver and of the kidney during lactation. Acta Physiologica Latin America, 1963, 13, 193-194.

Corbet, J. D. Effect of intravenous sodium chloride on drinking in the rat. Journal of Comparative and Physiological Psychology, $1965,60,397-406$.

Greizerstein, H. B., Abel, E. L., \& Siemens, A. J. Lactation and ethanol disappearance in the rat. Pharmacologist, 1979, $21,188$.

Greizenstein, H. B., \& Smith, C. M. Development and loss of tolerance to alcohol in goldfish. Journal of Pharmacology and Experimental Therapy, 1973, 187, 391-399.

Kennedy, G. C., Pearce, W. M., \& Parrott, D. M. V. Liver growth in the lactating rat. Journal of Endocrinology, 1958 , 17, 158-160.

Peters, J. M., \& Leeuwin, R. S. The effect of pregnancy and lactation on the pentobarbital-Na inducted sleeping-time in rats. Archives of International Pharmacodynamics and Therapy, 1971, 189, 370-372.

Thiessen, D. D., Whitworth, N., \& Rodgers, D. A. Reproductive functions and metabolic capacity as determinants of alcohol preference in $\mathrm{C}_{37} \mathrm{~B} 1$ female mice. Journal of Comparative and Physiological Psychology, 1967, 63, 151-154.

(Received for publication August 28, 1979.) 